\title{
Tumor Lysis Syndrome: A Rare but Serious Complication of Radioligand Therapies
}

\author{
Kai Huang ${ }^{1}$, Winfried Brenner ${ }^{1,2}$, and Vikas Prasad ${ }^{1,3}$ \\ ${ }^{I}$ Department of Nuclear Medicine, Charité-Universitätsmedizin Berlin, corporate member of Freie Universität Berlin, \\ Humboldt-Universität zu Berlin, and Berlin Institute of Health, Berlin, Germany; ${ }^{2}$ German Cancer Consortium, DKTK Partner \\ Site Berlin, Berlin, Germany; and ${ }^{3}$ Department of Nuclear Medicine, Universitätsklinikum Ulm, Ulm, Germany
}

Radioligand therapy (RLT) is considered a safe treatment for patients with metastasized neuroendocrine tumors and prostate cancer, and the occurrence of tumor lysis syndrome (TLS) with ${ }^{177} \mathrm{Lu}$-labeled peptides has not yet been reported. We retrospectively screened our patient database for TLS after RLT in neuroendocrine tumors and prostate cancer. Methods: The database was searched for patients receiving RLT with ${ }^{177}$ Lu-DOTATATE, -DOTATOC, or -prostate-specific membrane antigen and showing laboratory or clinical abnormalities typical of TLS within $7 \mathrm{~d}$ after the start of treatment. Results: In total, 205 patients (539 cycles) were screened; 4 patients developed TLS with clinical symptoms and characteristic changes in laboratory parameters, which normalized after appropriate treatment. Follow-up revealed partial remission in 2 patients, a mixed response in one, and progressive disease in one. Conclusion: Clinical TLS is a rare but definite complication of RLT, suggesting that patient monitoring for TLS should be mandatory.

Key Words: tumor lysis syndrome; radioligand therapy; neuroendocrine tumor; prostate cancer; ${ }^{177} \mathrm{Lu}$

J Nucl Med 2019; 60:752-755

DOI: 10.2967/jnumed.118.217380

$\mathbf{T}$ umor lysis syndrome (TLS), an oncometabolic emergency described in highly proliferating cancers such as lymphoma or leukemia, can occur spontaneously or after initiation of cytotoxic treatment such as chemotherapy or, rarely, radiation therapy or steroid treatment (1). Rapid tumor cell destruction leads to a massive release of intracellular contents such as potassium, phosphorus, and uric acid. This release may overload excretory mechanisms, leading to a clinicolaboratory derangement of cellular metabolism that finally results in acute renal failure (ARF), cardiac arrhythmia, central nervous system toxicity, and ultimately death (2). Serum calcium levels typically decrease because of binding of calcium to excess phosphorus (3).

Cairo et al. classified TLS based on laboratory and clinical criteria (4). Laboratory TLS is present if 2 or more of the relevant parameters-for example, uric acid, potassium, or phosphorusare increased or if calcium either is decreased by more than $25 \%$

Received Jul. 11, 2018; revision accepted Nov. 7, 2018.

For correspondence or reprints contact: Vikas Prasad, Department of Nuclear Medicine, Universitätsklinikum Ulm, Albert-Einstein-Allee 23, 89081 UIm, Germany.

E-mail: drvikaspd@yahoo.com

Published online Nov. 21, 2018.

COPYRIGHT (c) 2019 by the Society of Nuclear Medicine and Molecular Imaging. from baseline within $7 \mathrm{~d}$ after initiation of therapy or goes out of the reference range. For clinical TLS, the criteria are an increase of creatinine to more than 1.5 times the upper normal limit, an increase in blood urea nitrogen as a sign of renal failure, newly manifest cardiac arrhythmia, or seizures (4).

Radioligand therapies (RLTs) using ${ }^{177} \mathrm{Lu}$-labeled somatostatin receptor analogs or prostate-specific membrane antigen (PSMA) ligands are established and safe options for metastatic neuroendocrine tumors (NET) or prostate cancers (5-7). However, to our knowledge, TLS has never been reported for ${ }^{177} \mathrm{Lu}$-based RLT. In this study, we retrospectively evaluated the occurrence of TLS and its frequency in our patients.

\section{MATERIALS AND METHODS}

This study was approved by the institutional ethics review board (EA2/177/17). All patients signed a written informed consent form. The database of all patients undergoing RLT with ${ }^{177} \mathrm{Lu}$-DOTATATE, -DOTATOC, or -PSMA-617 between February 2011 and December 2017 and having complete patient records, including follow-up, was searched for prolonged hospitalization (more than the standard 48-h period), typical clinical complications (e.g., ARF, cardiac arrhythmia, or seizures within $7 \mathrm{~d}$ after RLT), typical laboratory constellations (e.g., hyperkalemia, hypocalcemia, hyperphosphatemia, uricemia, uremia, and increasing serum creatinine) (4), and clinical course during the immediate follow-up period.

RLTs were performed according to guidelines and recommendations as described in the literature (6-10). For nephroprotection, NET patients received amino acid solutions following the 3-d 50-g protection protocol (7); prostate cancer patients received $2 \mathrm{~L}$ of isotonic saline plus furosemide (9).

Serum potassium, blood urea nitrogen, uric acid, calcium, creatinine, and phosphorus were routinely measured by certified standard procedures by the university laboratory. RLT response based on imaging (PET, SPECT, CT, MRI) according to RESIST, version 1.1, or tumor markers (prostate-specific antigen, chromogranin A) at 2-3 mo after RLT was also recorded.

\section{RESULTS}

Of 539 RLTs performed on 205 patients with activities ranging from 4.9 to $7.5 \mathrm{GBq}$ of ${ }^{177} \mathrm{Lu}, 4$ patients $(0.74 \%$ of RLT cycles, $1.9 \%$ of patients) developed clinical TLS: 1 patient receiving DOTATATE (second cycle; pulmonary NET), 2 patients receiving DOTATOC (first and third cycles; pancreatic and unknown primary NET), and 1 patient receiving PSMA (first cycle; prostate cancer) (Table 1). One additional patient with prostate cancer developed a severe gout attack in both feet, revealing hyperuricemia and a distinct increase in uric acid. 
TABLE 1

Characteristics of Patients with TLS After RLT

\begin{tabular}{|c|c|c|c|c|c|c|c|c|}
\hline $\begin{array}{l}\text { Patient } \\
\text { no. }\end{array}$ & Sex & $\begin{array}{l}\text { Age } \\
(y)\end{array}$ & Diagnosis & Metastasis & Radioligand & $\begin{array}{l}\text { Cycle with } \\
\text { occurrence } \\
\text { of TLS }\end{array}$ & $\begin{array}{l}\text { Activity } \\
\text { (GBq) }\end{array}$ & Response \\
\hline $1^{*}$ & Male & 70 & Pulmonary NET & Bone, liver & 177Lu-DOTATATE & 2 & 5.0 & Mixed response \\
\hline $2^{\star}$ & Male & 66 & Pancreatic NET & Lymph node, spleen & 177Lu-DOTATOC & 3 & 7.5 & Partial remission \\
\hline $3^{\star}$ & Female & 79 & $\begin{array}{l}\text { NET of } \\
\text { unknown } \\
\text { primary }\end{array}$ & Liver, lymph node & 177Lu-DOTATOC & 1 & 4.9 & Partial remission \\
\hline $4^{\star}$ & Male & 75 & Prostate cancer & $\begin{array}{l}\text { Bone, lung, lymph } \\
\text { node, liver }\end{array}$ & ${ }^{177}$ Lu-PSMA & 1 & 6.0 & $\begin{array}{l}\text { Progressive } \\
\text { disease }\end{array}$ \\
\hline $5^{\dagger}$ & Male & 64 & Prostate cancer & Bone, lymph node & ${ }^{177 L u-P S M A}$ & 3 & 6.1 & Partial remission \\
\hline
\end{tabular}

Within $3 \mathrm{~d}$ after initiation of RLT, all 4 patients with clinical TLS went into ARF with increasing creatinine values (TLS grade 2 (4)), and a distinct increase in blood urea nitrogen was observed; 3 of these patients developed clinical symptoms such as disorientation and nausea within $24 \mathrm{~h}$. Uric acid in 3 patients met the criteria for TLS (Table 2). A calcium decrease was less prominent, with hypocalcemia occurring in 3 patients and 1 patient reported a tingling paraesthesia of the hands. Mild hyperkalemia was observed in 2 patients at $48 \mathrm{~h}$. None of the patients developed cardiac arrhythmia or seizures.

Patients were treated symptomatically according to TLS guidelines (11). Patients with ARF received vigorous intravenous hydration with isotonic solutions. Furosemide in a dose of $0.5 \mathrm{mg} / \mathrm{kg}$ of body weight was administered to increase excretion. Allopurinol was prescribed for uricemia, and rasburicase was given intravenously to 1 patient. For treatment of the gout attack, ibuprofen and celecoxib in combination with colchicine were prescribed. In cases of symptomatic hypocalcemia, calcium was substituted orally at a dosage of up to $1.5 \mathrm{~g} / \mathrm{d}$.

All patients were able to leave the hospital within $7 \mathrm{~d}$ without clinical symptoms and with normalized or near-normal laboratory parameters.

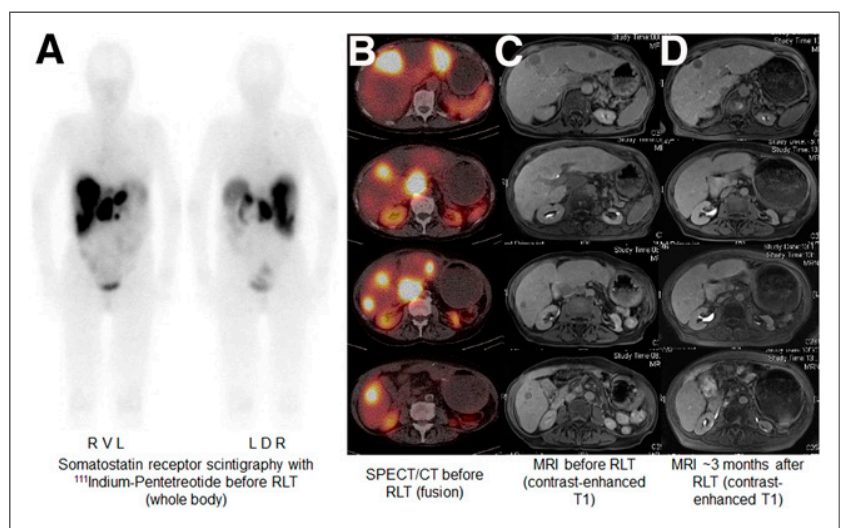

FIGURE 1. Images of 79-y-old woman with NET of unknown primary G1 $(\mathrm{Ki}-67=2 \%)$ and multiple liver and lymph node metastases showing partial remission after 1 cycle of RLT with $4.972 \mathrm{GBq}$ of ${ }^{177} \mathrm{Lu}$-DOTATOC. TLS occurred $3 \mathrm{~d}$ after RLT, with ARF, disorientation, and nausea. $\mathrm{LDR}=$ left, dorsal, right; $\mathrm{RVL}=$ right, ventral, left.
Follow-up revealed partial remission in 2 TLS patients, a mixed response in 1 patient, and progressive disease in 1 patient (Table 1; Fig. 1). The patient with a mixed response had additional bone lesions on PET without a morphologic correlation, but the target lesions showed a clear response.

\section{DISCUSSION}

We observed TLS after RLT in $1.9 \%$ of patients and in $0.74 \%$ of RLT cycles, and all 4 patients developed ARF. Historically, RLT is well tolerated and high-grade nephrotoxicity occurs in less than $1 \%$ of patients, with a delay of months to years $(6,12,13)$. The occurrence of ARF due to TLS after RLT with ${ }^{90}$ Y-DOTATOC has been reported in 2 of 1,109 patients (14). However, to our knowledge TLS after treatment with ${ }^{177} \mathrm{Lu}$-labeled peptides has not yet been reported. None of our patients received other cytotoxic treatments within 4 wk of RLT. Therefore, TLS most probably was caused by ${ }^{177} \mathrm{Lu}$-based RLT. The recommended treatment for TLS was successfully started directly after indicated by laboratory abnormalities or after the onset of clinical symptoms in these patients $(4,11)$. To prevent or minimize TLS, appropriate patient preparation, specific laboratory tests (creatinine, blood urea nitrogen, uric acid, potassium, calcium, phosphate), and a

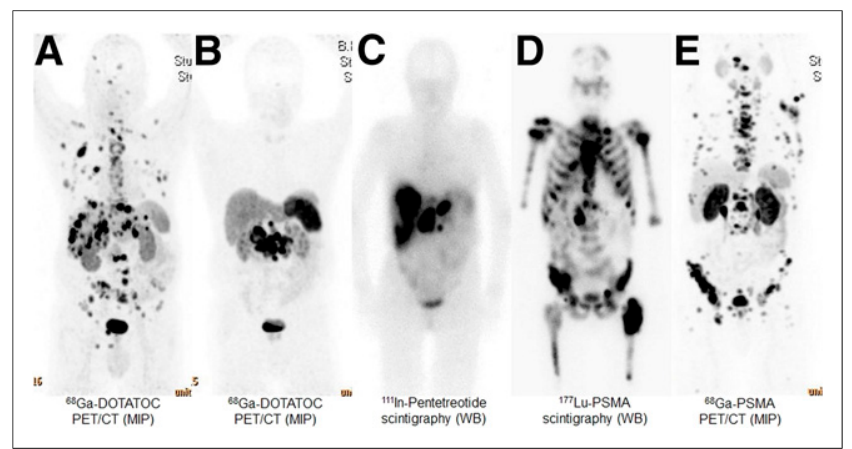

FIGURE 2. Tumor load on pretherapeutic and intratherapeutic images of all 4 TLS patients (A-D) and of patient with severe gout attack (E). MIP = maximum-intensity projection; $\mathrm{WB}=$ whole body. 


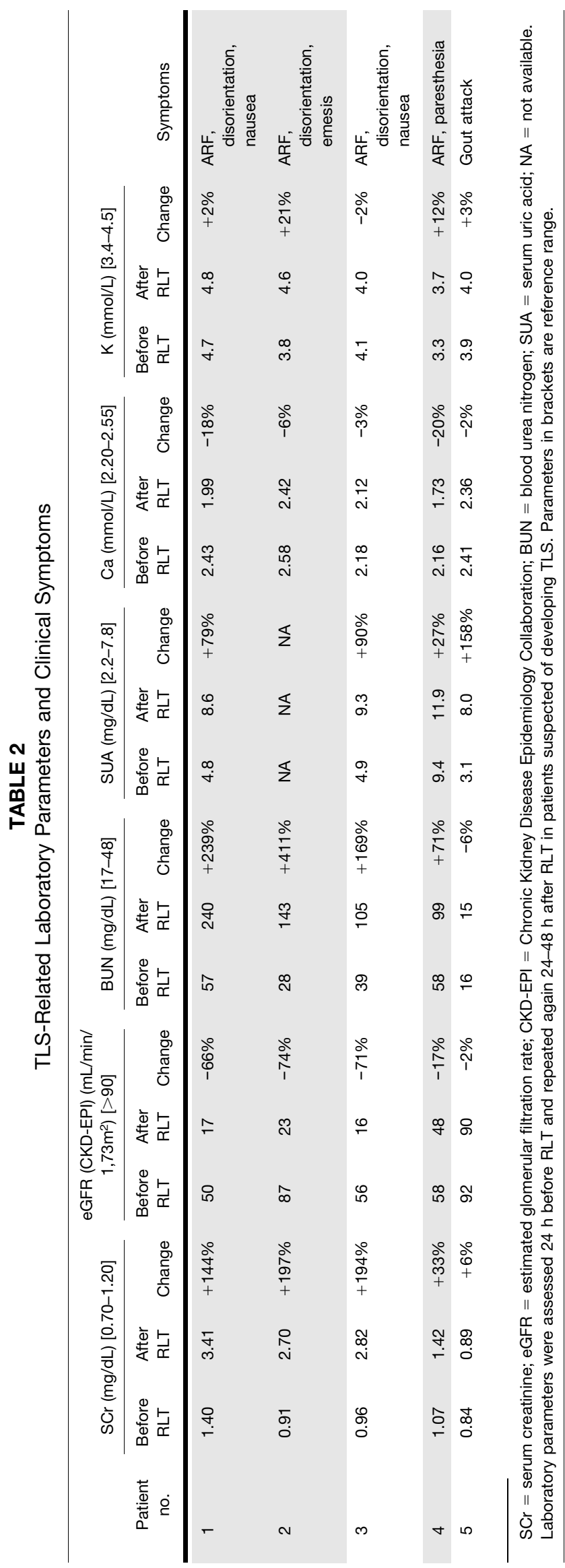

history of chronic renal or cardiac diseases should be established in all patients scheduled for ${ }^{177} \mathrm{Lu}$-based RLT. One of the strategies to prevent TLS after RLT is to intensively hydrate (infusion of 1-2 L of isotonic solution per day) patients with elevated serum creatinine or a very high tumor burden (assessed visually) starting $1 \mathrm{~d}$ before RLT and continuing until discharge from the hospital. Prophylactic treatment with allopurinol, 100-300 mg/d, may be started if the uric acid level is elevated or in the upper reference range. If hypocalcemia is detected before RLT, calcium should be substituted orally. In severe cases of ARF, hemodialysis may be considered (4). Because of the low number of patients, we did not statistically test for predisposing factors for TLS, such as age, sex, tumor burden, pretreatment, or laboratory parameters. Our TLS patients were relatively old, ranging from 64 to $79 \mathrm{y}$, whereas the age range for all RLT patients was $45-83 \mathrm{y}$.

TLS was observed after the first, second, or third cycle of RLT in different patients for both ${ }^{177} \mathrm{Lu}$-PSMA and ${ }^{177} \mathrm{Lu}$-DOTATATE/ DOTATOC. The amount of radioactivity had no relevant impact: even patients treated with reduced radioactivity developed TLS. Pretherapeutic laboratory parameters were rather inhomogeneous, with values ranging from normal to marginally pathologic (Table 2). The extent of tumor burden was also not predictive in these few patients, as both patients with very high and patients with very low tumor burden developed TLS (Fig. 2). Future research is needed to assess patient- or tumor cell-specific characteristics that might predispose a patient to TLS (15).

TLS patients benefited from RLT: 2 of 4 patients with ARF achieved partial remission within 2-3 mo, and 1 patient showed a mixed response. Because partial remission due to RLT is reportedly in the range of $30 \%-40 \%$ (16), TLS patients do not seem to do worse than other patients, as has been shown for chemotherapy in multiple myeloma (17).

The major limitation of this study, besides the low number of TLS patients, is that we did not routinely perform all relevant TLS-specific laboratory parameters after RLT. Thus, the true percentage of patients developing laboratory TLS may be higher than observed. However, laboratory TLS only, with absence of clinical symptoms, is not a life-threatening event in general.

\section{CONCLUSION}

Symptomatic TLS after RLT seems very rare but can lead to severe symptoms, including ARF. Because predictive parameters for TLS in RLT are not yet known, it is important to assess and prepare patients before and after RLT by taking precautionary measures with respect to laboratory parameters-for example, allopurinol, hydration, and calcium substitution-and to keep in mind that TLS may occur after ${ }^{177}$ Lu-based RLT.

\section{DISCLOSURE}

No potential conflict of interest relevant to this article was reported.

\section{REFERENCES}

1. Abu-Alfa AK, Younes A. Tumor lysis syndrome and acute kidney injury: evaluation, prevention, and management. Am J Kidney Dis. 2010;55:S1-13.

2. Mirrakhimov AE, Voore P, Khan M, Ali AM. Tumor lysis syndrome: a clinical review. World J Crit Care Med. 2015;4:130-138.

3. Rampello E, Fricia T, Malaguarnera M. The management of tumor lysis syndrome. Nat Clin Pract Oncol. 2006;3:438-447. 
4. Cairo MS, Bishop M. Tumour lysis syndrome: new therapeutic strategies and classification. Br J Haematol. 2004;127:3-11.

5. Strosberg J, El-Haddad G, Wolin E, et al. Phase 3 trial of ${ }^{177} \mathrm{Lu}$-dotatate for midgut neuroendocrine tumors. N Engl J Med. 2017;376:125-135.

6. Rahbar K, Ahmadzadehfar H, Kratochwil C, et al. German multicenter study investigating ${ }^{177} \mathrm{Lu}$-PSMA-617 radioligand therapy in advanced prostate cancer patients. J Nucl Med. 2017;58:85-90.

7. Bodei L, Mueller-Brand J, Baum RP, et al. The joint IAEA, EANM, and SNMMI practical guidance on peptide receptor radionuclide therapy (PRRNT) in neuroendocrine tumours. Eur J Nucl Med Mol Imaging. 2013;40:800-816.

8. Hicks RJ, Kwekkeboom DJ, Krenning E, et al. ENETS consensus guidelines for the standards of care in neuroendocrine neoplasia: peptide receptor radionuclide therapy with radiolabeled somatostatin analogues. Neuroendocrinology. 2017;105: 295-309.

9. Fendler WP, Kratochwil C, Ahmadzadehfar H, et al. Nucl Med (Stuttg). ${ }^{177} \mathrm{Lu}-$ PSMA-617 therapy, dosimetry and follow-up in patients with metastatic castration-resistant prostate cancer [in German]. 2016;55:123-128.

10. Kwekkeboom DJ, Krenning EP, Lebtahi R, et al. ENETS consensus guidelines for the standards of care in neuroendocrine tumors: peptide receptor radionuclide therapy with radiolabeled somatostatin analogs. Neuroendocrinology. 2009;90: 220-226.
11. Jones GL, Will A, Jackson GH, Webb NJ, Rule S; British Committee for Standards in Haematology. Guidelines for the management of tumour lysis syndrome in adults and children with haematological malignancies on behalf of the British Committee for Standards in Haematology. Br J Haematol. 2015;169:661-671.

12. Ahmadzadehfar H, Rahbar K, Kurpig S, et al. Early side effects and first results of radioligand therapy with ${ }^{177} \mathrm{Lu}-\mathrm{DKFZ}-617$ PSMA of castrate-resistant metastatic prostate cancer: a two-centre study. EJNMMI Res. 2015;5:114.

13. Baum RP, Kluge AW, Kulkarni H, et al. $\left[{ }^{177} \mathrm{Lu}-\mathrm{DOTA}\right]^{0}$-D-Phe ${ }^{1}-\mathrm{Tyr}^{3}$-Octreotide $\left({ }^{177} \mathrm{Lu}-\mathrm{DOTATOC}\right)$ for peptide receptor radiotherapy in patients with advanced neuroendocrine tumours: a phase-II study. Theranostics. 2016;6:501-510.

14. Imhof A, Brunner P, Marincek N, et al. Response, survival, and long-term toxicity after therapy with the radiolabeled somatostatin analogue [ $\left.{ }^{90} \mathrm{Y}-\mathrm{DOTA}\right]-$ TOC in metastasized neuroendocrine cancers. J Clin Oncol. 2011;29:2416-2423.

15. Hennequin C, Quero L, Favaudon V. Determinants and predictive factors of tumour radiosensitivity [in French]. Cancer Radiother. 2008;12:3-13.

16. Ahmadzadehfar H, Essler M. Predictive factors of response and overall survival in patients with castration-resistant metastatic prostate cancer undergoing ${ }^{177} \mathrm{Lu}-$ PSMA therapy. J Nucl Med. 2018;59:1033-1034.

17. Chim CS. Rapid complete remission in multiple myeloma with bortezomib/ thalidomide/dexamethasone combination therapy following development of tumor lysis syndrome. Cancer Chemother Pharmacol. 2008;62:181-182. 